\title{
Quantitative Reagent-Free Detection Of Fibrinogen Levels In Human Blood Plasma Using Raman Spectroscopy
}

\author{
Kelvin Poon \\ Technological University Dublin \\ Fiona Lyng \\ Technological University Dublin, fiona.lyng@tudublin.ie \\ Peter Knief \\ Technological University Dublin
}

See next page for additional authors

Follow this and additional works at: https://arrow.tudublin.ie/radart

Part of the Biochemistry, Biophysics, and Structural Biology Commons, Biological and Chemical Physics Commons, Biotechnology Commons, and the Medical Biotechnology Commons

\section{Recommended Citation \\ Poon, K.W. C., Lyng, F. M., Knief, P., Howe, O., Meade, A. D., Curtin, J. F., Byrne, H. J., and Vaughan, J., "Quantitative reagent-free detection of fibrinogen levels in human blood plasma using Raman spectroscopy", The Analyst, 137, 1807-1814 (2012). doi:10.1039/C2AN35042D}

This Article is brought to you for free and open access by the Radiation and Environmental Science Centre at ARROW@TU Dublin. It has been accepted for inclusion in Articles by an authorized administrator of ARROW@TU Dublin. For more information, please contact arrow.admin@tudublin.ie, aisling.coyne@tudublin.ie, gerard.connolly@tudublin.ie. Funder: HEA PRTLI Cycle 4 NBIPI

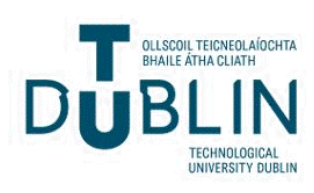




\section{Authors}

Kelvin Poon, Fiona Lyng, Peter Knief, Orla L. Howe, Aidan Meade, James Curtin, Hugh Byrne, and Joseph Vaughan 


\title{
Quantitative reagent-free detection of fibrinogen levels in human blood plasma using Raman spectroscopy
}

\author{
Kelvin W. C. Poon, ${ }^{a}{ }^{a}$ Fiona M. Lyng, ${ }^{a}$ Peter Knief, ${ }^{b}$ Orla Howe, ${ }^{a, b}$ Aidan D. Meade, ${ }^{b}$ James F. Curtin, ${ }^{c}$ \\ Hugh J. Byrne, ${ }^{b}$ and Joe Vaughan ${ }^{c}$
}

\author{
${ }_{5}$ Received (in XXX, XXX) Xth XXXXXXXXX 20XX, Accepted Xth XXXXXXXXX 20XX \\ DOI: $10.1039 / b 000000 x$
}

Fibrinogen assays are commonly used as part of clinical screening tests to investigate haemorrhagic states, for detection of disseminated intravascular coagulation and as a predictor of a variety of cardiovascular events. The Clauss assay, which measures thrombin clotting time, is the most commonly

10 used method for measuring fibrinogen levels. Nevertheless, inconsistencies are present in intermanufacturer reagent sources, calibration standards and methodologies. Automated coagulation analysers, which measure changes in optical density during the prothrombin time (PT-Fg), have found use in many hospitals. However, the PT-Fg method is found to give falsely elevated values due to varying choices of calibrants, reagents and analysers. As an alternative, Raman spectroscopy has previously been applied to 15 the analysis of blood and its various constituents to determine various analyte concentrations such as glucose, urea, triglycerides and cholesterol. In this study, Raman spectroscopy was investigated for its ability to accurately quantify fibrinogen concentration in blood plasma. Samples collected from 34 patients were analysed by Raman spectroscopy and the resultant spectra were fitted with a Partial Least Squares Regression model using target values obtained through a pre-calibrated Clauss fibrinogen assay.

${ }_{20}$ Various spectral pre-processing methods were utilised to prepare data to be entered into a calibration model. A root mean square error of prediction of $0.72 \pm 0.05 \mathrm{~g} / \mathrm{L}$ was achieved with as few as 25 spectra. In this pilot study, Raman spectroscopy has been demonstrated to be a robust technique providing rapid and reagent-free quantification of fibrinogen levels in blood plasma and a potential alternative to the Clauss assay.

\section{${ }_{25}$ Introduction}

The activation of fibrinogen or Factor I, a $340 \mathrm{kDa}$ dimeric plasma glycoprotein synthesised in the hepatocytes of the liver, is involved as an acute phase protein in the pathogenesis of inflammation, tumour growth and various diseases. During 30 circulation, fibrinogen is degraded into various molecular weights, $69.7 \%$ remaining in the original high molecular weight (HMW) form (340 kDa), $26.5 \%$ as low molecular weight (LMW') fibrinogens $(305 \mathrm{kDa})$ and the remaining $3.8 \%$ in the low molecular weight (LMW) configuration of $270 \mathrm{kDa}$, as a 35 result of the consecutive removal of a $35 \mathrm{kDa}$ carboxyterminal polypeptide from one of the A $\alpha$-chains. ${ }^{1}$ Epidemiological studies have demonstrated elevated levels of fibrinogen in blood to be an independent risk factor for cardiovascular and coronary heart disease $^{2,3}$, while low fibrinogen levels are seen as an important 40 diagnostic physiological variable in pathological conditions including various haemorrhagic states, disseminated intravascular coagulation, liver disease and during thrombolytic/defibrination therapy. ${ }^{4}$ Fibrinogen concentration has also been shown to be an independent prognostic parameter in patients diagnosed with 45 cervical cancer. ${ }^{5}$

Fibrinogen levels in plasma may be determined through various methods. These methods are based on different techniques of detection such as measures of clotting rate ${ }^{6}$ or clottable protein, ${ }^{7}$ turbidimetry (light scattering of the sample), ${ }^{8}$ 50 immunological/antibody ${ }^{9}$ and heat $/$ salt precipitation ${ }^{10}$. Assays based on clotting rates and changes in turbidity/light-scattering are most commonly used in clinical practice. The Clauss fibrinogen assay, developed in 1957, measures the thrombin clotting time and remains the most frequently used technique for ${ }_{55}$ fibrinogen measurement. The method entails the addition of excess thrombin to diluted plasma samples and the subsequent measurement of the clotting time. The recorded time is compared with a calibration curve constructed through the measurement of clotting times from a series of dilutions of reference plasmas of 60 known fibrinogen concentration, allowing the concentration in the test sample to be established. Prothrombin time-derived (PT$\mathrm{Fg}$ ) methods are currently used in approximately $50 \%$ of UK Haematology laboratories ${ }^{11}$, their popularity being largely due to the introduction of coagulation automates. The assay measures ${ }_{65}$ the turbidity and light-scattering properties after the complete clotting of the plasma occurs. The analyser requires calibration through the recording of the prothrombin time on a series of plasma dilutions of known fibrinogen concentration and the subsequent construction of a graph of the change in optical 70 properties against fibrinogen concentration. The optical response recorded from the test sample may then be converted to fibrinogen content. It has also been shown that the LMW and LMW' fibrinogens exhibit prolonged clotting times compared with HMW fibrinogen. ${ }^{12}$

75 Despite the popularity of the two assays, several issues have been raised regarding their reproducibility and standardisation. The Clauss assay is relatively time consuming with respect to calibration, requiring sufficient expertise to evaluate difficult endpoints from high dilutions of standard plasmas, samples with low 80 fibrinogen content or those forming unstable clots. ${ }^{4}$ Analysers 
relying on mechanical, tensile clot strength end-point detection may be affected by heparin therapy while photo-optical systems measuring light scattering changes may be vulnerable to slow fibrin polymerisation, turbid reference plasma samples along with 5 the presence of any bile pigment or free haemoglobin., 43 Inconsistencies arise when fibrinogen levels are measured using commercial reagents sourced from different manufacturers. For example, thrombin strength, buffer composition (anticoagulant inhibitors and degradation products) along with calibration and 10 dilution methods suggested by the various manufacturers vary significantly. ${ }^{14}$ Despite the availability of International Standard preparations for plasma fibrinogen, some commercial reference samples have been found to be incorrectly calibrated using internal laboratory plasma samples or their respective national 15 standards. ${ }^{15}$ Substantial differences were also observed depending on the type of analyser used.

Raman spectroscopy measures vibrational modes in molecules and can be employed to characterise more complex mixtures of molecules. The spectrum of a complex biological mixture of 20 proteins is composed of contributions from each of the major components in that mixture, and changes in the concentration of one or more components in that mixture will give rise to reproducible changes in the Raman spectrum. Both Raman and infrared spectroscopy, in combination with multivariate statistical 25 techniques, have previously been investigated as a potential clinical diagnostic tool to differentiate between normal and malignant cells ${ }^{16-18}$ as well as tissues from the skin, breast, prostate, oesophagus and cervix. ${ }^{19-23}$ Furthermore, it has been demonstrated that vibrational spectroscopy has the ability to 30 quantify various analyte concentrations (total protein, glucose, urea, triglycerides and cholesterol etc.) in blood and its fluid derivatives. $^{24-32}$ In contrast to current assay approaches, which rely on physicochemical means of determining fibrinogen, vibrational spectroscopy provides detailed chemical information 35 of the biological compounds, each possessing unique spectral signatures. Rohleder et al. compared mid-infrared spectroscopy to Raman spectroscopy with respect to the analysis of various blood serum constituents including total protein content ${ }^{24}$ and the strengths and weaknesses of each technique were addressed 40 (sample preparation, operational parameters etc.). Significantly it was found that Raman spectroscopy can quantify the total protein content with lower root mean square errors of prediction than corresponding mid-infrared spectroscopic results. While Raman spectroscopy has been used previously to establish total protein 45 content in blood serum, ${ }^{24,31,33}$ the current pilot study attempts to distinguish and quantify the single component of fibrinogen in dried, undiluted, whole human blood plasma collected from patients. Being able to determine changes in protein levels in whole blood samples compared to serum has the potential 50 advantage of minimising sample processing. Although fibrinogen is a minor component of the total protein content $(\sim 4 \%)$, the ability to correlate sample dependent variations in content, through a regression model relating changes in spectroscopic signatures to the gold standard Clauss assay, promises a potential 55 alternative routine screening assay. Spectral data was therefore collected and modelled using a partial least squares regression algorithm, correlating values to those obtained in parallel using a pre-calibrated Clauss Fibrinogen assay.

\section{EXPERIMENTAL PROCEDURES}

\section{${ }_{60}$ Blood Plasma Preparation}

Venous blood from 34 patients was collected in BD Vacutainer ${ }^{\mathrm{TM}}$ Plus plastic tri-sodium citrate tubes. After eight inversions, each tube was centrifuged at $1500 \mathrm{~g}$ for 15 minutes to separate the plasma and cellular components. Samples were immediately 65 stored at $-20{ }^{\circ} \mathrm{C}$ until needed. Prior to measurement, the microtubes were thawed in a $37{ }^{\circ} \mathrm{C}$ water bath and $10 \mu \mathrm{L}$ of plasma carefully spotted in $0.75 \times 0.75 \mathrm{~cm}^{2}$ squares onto precleaned calcium fluoride windows (Crystran Ltd., UK).

Table 1. Performance of Clauss Fibrinogen Assay (HemosIL ${ }^{\circledR}$ 70 Fibrinogen-C ACL Top Family)

\begin{tabular}{lccc}
\hline & Sample Number & Range $(\mathrm{g} / \mathrm{L})$ & \\
\hline Normal Range & 119 & $2.38-4.98$ & \\
\hline & $\begin{array}{c}\text { Mean Fibrinogen } \\
\mathrm{g} / \mathrm{L})\end{array}$ & $\begin{array}{c}\mathrm{CV} \% \\
\text { (run to run) }\end{array}$ & $\begin{array}{c}\mathrm{CV} \% \\
\text { (day to day) }\end{array}$ \\
\hline Normal Control & 3.03 & 4.5 & 5.5 \\
Low Control & 1.07 & 5.1 & 6.8 \\
Linearity & $0.35-10$ & & \\
\hline
\end{tabular}

The plasma squares were then passively dried at room temperature for at least 4 hours in a desiccator filled with a silica gel desiccant. Fibrinogen content was determined using a pre75 calibrated HemosIL $\AA$ Fibrinogen-C ACL Top Family Clauss assay; performance values provided by the manufacturer are listed in Table 1. The fibrinogen content recorded from patient samples ranged from 0.60 to $8.18 \mathrm{~g} / \mathrm{L}$. Crystalline purified Fibrinogen from human plasma, used as a comparative control for 80 the spectral measurements, and was purchased from Sigma-

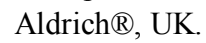

\section{Instrumentation}

\section{Raman Measurements}

Raman spectra were recorded on a Horiba Jobin-Yvon ${ }_{85}$ (Villeneuve d'Ascq, France) HR800 LabRam Raman spectrometer (LabSpec V5.0 software) using a $785 \mathrm{~nm}$ diode laser source $(\sim 110 \mathrm{~mW}$ at the sample), focussed onto the sample through a $100 \mathrm{x}$ objective lens (NA=0.9, MPlan, Olympus). Raman scatter was collected in a back-scattered configuration ${ }_{90}$ through a $100 \mu \mathrm{m}$ confocal aperture onto a spectrograph/Peltiercooled Synapse CCD detector. A 300 line $/ \mathrm{mm}$ grating was used to scan a spectral region between $400-1800 \mathrm{~cm}^{-1}$, achieving a dispersion of $\sim 1.5 \mathrm{~cm}^{-1}$ per pixel. Calibration was performed with reference to the $520.7 \mathrm{~cm}^{-1}$ peak of crystalline $\mathrm{Si}$, and spectra 95 were corrected for detector dark noise, system optics and system spectral response through the use of a Raman Intensity Correction Standard (SRM 2241, NIST). Spectra were recorded from a square array of $10 \times 10$ points (totalling 100) spaced $2 \mu \mathrm{m}$ apart using $30 \times 3$ second acquisition periods per point. The array was 100 collected from the homogenous middle of the dried plasma square.

\section{FTIR Measurements}

Parallel infrared absorption spectroscopic imaging was carried out using the same dried plasma squares on $\mathrm{CaF}_{2}$ windows on a 105 Perkin Elmer (Waltham, Massachusetts, USA) Spotlight 400N FTIR imaging system equipped with a linear array of 16 liquid nitrogen cooled MCT detectors. A $25 \mu \mathrm{m}$ x $25 \mu \mathrm{m}$ pixel 
resolution was used to capture an average absorption image in transmission mode.

\section{Data Analysis}

A host of pre-processing techniques were trialled in various 5 combinations on the raw dataset to produce an equally weighted input into the regression model for reducing prediction errors. Methods included combinations of a Savitzky-Golay smoothing filter, vector normalisation, linear/polynomial baseline offset correction and extended multiplicative scatter correction. All pre10 processing methods were performed using Matlab ${ }^{\text {TM }}$ V7.7 (Mathworks, Natick, Massachusetts) in conjunction with PLS Toolbox 5.22 (Eigenvector Research, Wenatchee, WA).

A common method for creation of calibration models for prediction of target analyte concentrations with vibrational 15 spectra is Partial Least Squares Regression (PLSR). ${ }^{29,} 31,34,35$ The PLS model attempts to elucidate factors or latent variables $(\mathrm{LV})$ which account for the systematic majority of variation in predictors ' $\mathbf{X}$ ' (spectral data) versus associated responses ' $Y$ ' (target values of fibrinogen concentration). In other words, PLS 20 uses the information contained in $\mathbf{Y}$ to determine the systematic multivariate signatures in $\mathbf{X}$. This is achieved through the reduction of the variables corresponding to the wavenumber axis of the spectral data $\mathbf{X}$ into reduced number of spectral variables while simultaneously reducing variables containing associated 25 target concentration information $\mathbf{Y}$ in each training sample. Latent variables that are best correlated with variations in target values allow the creation of a calibration model that can subsequently be tested using test data. The linear regression model is created by reducing the $\mathbf{X}$ and $\mathbf{Y}$ matrices into latent

30 variables and scores via principal component analysis (PCA) such that the $\mathbf{X}$ matrix scores are regressed against the $\mathbf{Y}$ matrix scores in the model. The process can therefore be described mathematically as:

$$
\begin{aligned}
\mathbf{X} & =\mathrm{TP}^{\prime}+\mathrm{E} \\
{ }_{35} \mathbf{Y} & =\mathrm{UQ}^{\prime}+\mathrm{F}
\end{aligned}
$$

where; $\mathbf{X}$ and $\mathbf{Y}=$ Matrices of the predictors and responses respectively.

$\mathrm{T}=$ Matrix of $\mathrm{X}-$ scores

$\mathrm{U}=$ Matrix of $\mathrm{Y}-$ scores

40 $\mathrm{P}=$ Matrix of $\mathrm{X}$ - latent variables

$\mathrm{Q}=$ Matrix of $\mathrm{Y}$ - latent variables

$\mathrm{E}=\mathrm{X}-$ residual errors

$\mathrm{F}=\mathrm{Y}-$ residual errors

Cross validation is a technique enabling the assessment of the 45 optimal complexity of a model (in this case the number of latent variables in the PLS model) thus allowing an estimation of the performance of a model when applied to unknown data. Cross validation involves a series of sub-validation experiments, which see the removal of a subset of objects (or spectra) from a dataset 50 for testing (the test set), construction of a model using the remaining objects in the dataset (the calibration set), and subsequent application of the resulting model to the test set. This ensures each sub-validation experiment robustly tests a model using objects which are independent of those used to construct ${ }_{55}$ the model. In this study, two cross validation methods were used for comparative purposes: Random sample cross validation, which chooses different random subset test sets determined through the random selection of $n / s$ objects in the data set (where $n$ is the total number of objects in the dataset and $s$ is the ${ }_{60}$ predetermined number of data splits), such that no single object appears in more than one test set. This procedure is repeated for a user defined number of iterations. In this study, a split of $s=5$ was chosen and the process was iterated 100 times. The second method is Leave-One-Out cross validation, involves the use of a ${ }_{65}$ single element from the data set being used as a test or validation 'set' while the remaining objects are used as the training set. This process is iteratively repeated so that every single observation in the data set is used once as the designated validation data set.

70 The SIMPLS algorithm ${ }^{36}$ of the PLS Toolbox v5.22 (Eigenvector Research, Inc.) was implemented within Matlab. The training of the teaching data set was optimised by calculating the root-meansquare error of calibration (RMSEC) and the root-mean-square error of cross validation (RMSECV). The spectral data obtained 75 from the 33 samples were split 60/40 into training and test sets respectively. The various RMSE were calculated according to equations 3, 4 and 5 below:

$\mathrm{RMSEC}=\sqrt{\frac{\sum_{i=1}^{N_{\text {train }}}\left(x_{\text {pred }, i}-y_{\text {resp }, i}\right)^{2}}{N_{\text {train }}-L V-1}}$

80

$\mathrm{RMSECV}=\sqrt{\frac{\sum_{i=1}^{N_{\text {train }}}\left(x_{\text {pred }, i}-y_{\text {resp }, i}\right)^{2}}{N_{\text {train }}}}$

$\mathrm{RMSEP}=\sqrt{\frac{\sum_{i=1}^{\text {Ntest }}\left(x_{\text {pred }, i}-y_{\text {resp }, i}\right)^{2}}{N_{\text {test }}}}$

Here, $x_{\text {pred }, i}$ and $y_{\text {resp }, i}$ correspond to fibrinogen content in sample $i$ 85 determined by Raman spectral data and the Clauss assay, respectively. $N_{\text {train }}$ and $N_{\text {test }}$ denotes the number of training $\left(N_{\text {train }}\right.$ $=20 \times n$ spectra $)$ and test samples $\left(N_{\text {test }}=14 \times n\right.$ spectra $)$ respectively. The number of latent variables used for the PLS calibration is optimised by finding the value that corresponds to 90 the minimum of the RMSECV. Using the training values, a model is constructed and the quality of fit is judged by how well 
Table 2. Peak assignments ${ }^{37}$ for the Raman spectra of blood plasma.

\begin{tabular}{|c|c|}
\hline Peak Position $\left(\mathrm{cm}^{-1}\right)$ & Assignments of Raman Vibrational Modes \\
\hline 540 & Histidine $^{\mathrm{a}}$ \\
\hline 621 & $\mathrm{C}-\mathrm{C}$ twisting mode of phenylalanine \\
\hline 642 & $\mathrm{C}-\mathrm{C}$ twisting mode of tyrosine \\
\hline 758 & Symmetric ring breathing of tryptophan \\
\hline 829 & Out of plane ring breathing tyrosine \\
\hline 853 & Ring breathing mode of tyrosine \\
\hline 939 & $\mathrm{C}-\mathrm{C}$ stretching mode backbone ( $\alpha$-helix conformation) \\
\hline 958 & $\mathrm{CH}_{3}$ deformation \\
\hline 1003 & Symmetric ring breathing mode of phenylalanine \\
\hline 1032 & $\mathrm{C}-\mathrm{H}$ in-plane bending mode of phenylalanine \\
\hline 1082 & $\mathrm{C}-\mathrm{N}$ stretching mode \\
\hline 1128 & $\mathrm{C}-\mathrm{N}$ stretching mode \\
\hline 1158 & $\mathrm{C}-\mathrm{C} / \mathrm{C}-\mathrm{N}$ stretching mode \\
\hline 1208 & Tryptophan and phenylalanine $\mathrm{n}\left(\mathrm{C}-\mathrm{C}_{6} \mathrm{H}_{5}\right)$ mode \\
\hline 1271 & Amide III ( $\mathrm{C}-\mathrm{N}$ stretching mode, mainly a-helix conformation) \\
\hline 1318 & $\mathrm{C}-\mathrm{H}$ deformation \\
\hline 1339 & $\mathrm{C}-\mathrm{H}$ deformation \\
\hline 1447 & $\mathrm{C}-\mathrm{H}$ deformation \\
\hline 1552 & Tryptophan \\
\hline 1585 & $\mathrm{C}=\mathrm{C}$ stretching \\
\hline 1606 & $\mathrm{C}=\mathrm{C}$ stretching mode of phenylalanine and tryptophan \\
\hline 1616 & $\mathrm{C}=\mathrm{C}$ stretching mode of tyrosine and tryptophan \\
\hline 1659 & Amide $\mathrm{I}(\mathrm{C}=\mathrm{O}$ stretching mode of proteins, a-helix conformation) \\
\hline
\end{tabular}

${ }^{a}$ Tentative assignments.

it is able to predict the values from the test data set. The rootmean-squared error of calibration (RMSEC) and the root-mean5 squared error of prediction (RMSEP) are used to evaluate the predictive capacity of the constructed model. The efficacies of the various pre-processing and validation methods were evaluated through the performance of the PLS model.

\section{RESULTS}

${ }_{10}$ While water possesses a low Raman scattering cross section in the visible region, it is resonantly enhanced in the near infrared due to the onset of O-H vibrational overtones. ${ }^{38}$ Furthermore, as it has only been mildly centrifuged, the protein rich blood plasma scatters significantly, introducing a large background to the 15 spectrum which swamps the Raman signal of the solute. ${ }^{39}$ Therefore dried samples were chosen over fresh/wet samples. However, the drying process resulted in inhomogeneous samples as constituents precipitated predominantly at the edge of the spotted square either through surface tension or molecular weight 20 differences. Figure 1 shows an FTIR image illustrating this sample inhomogeneity in terms of spectrally averaged absorbance. These variable thicknesses produced Raman spectra of various intensities and backgrounds due to differences in sample density cross-sections.

${ }_{25}$ To maintain a level of consistency, Raman spectra were always recorded from the middle of the dried spot as it was the largest area of consistent thickness and uniformity. Figure 2 shows the mean Raman spectrum of dried blood plasma (top) (smoothed, baseline corrected and normalised) together with a spectrum of 30 crystalline purified Fibrinogen from human plasma (bottom) (Sigma-Aldrich $\AA$, UK) for spectral comparison. Total plasma proteins (albumin and globulin) are the most abundant species in blood plasma and hence spectra are characteristic of these protein base vibrations. The observed peaks primarily show the
35 tyrosine/phenylalanine twisting doublet at $621 / 642 \mathrm{~cm}^{-1}$ while the tyrosine ring-breathing doublet can be seen at $829 / 860 \mathrm{~cm}^{-1}$. The sharpest and most dominant peak at $1003 \mathrm{~cm}^{-1}$ is attributed to the phenylalanine symmetric breathing mode while $\mathrm{CH}_{2}$ bending appears at $1446 \mathrm{~cm}^{-1}$ and the $\mathrm{C}=\mathrm{O}$ stretching mode of Amide I at $401657 \mathrm{~cm}^{-1}$. The Raman spectrum of crystalline fibrinogen contains similar features, but notably has strong contributions from tryptophan at $758 \mathrm{~cm}^{-1}$ and $1552 \mathrm{~cm}^{-1}$. This is surprising as fibrinogen does not have a high tryptophan content, but may be due to sample impurities or alignment of tryptophan moieties in

${ }_{45}$ the crystalline sample. A full list of peak assignments is provided in Table 2.

\section{Spectral Pre-processing}

Despite the improvements over the low signal-to-noise limitations associated with the analysis of wet plasma samples, 50 the dried plasma films still exhibited inconsistencies evident in the variable backgrounds seen in the raw spectral data (Figure 3). Although fluorescence is a possible origin of such backgrounds, the collection of blood plasma involves gradient and centrifugation separation, which should have removed all cellular 55 material, including red blood cells and therefore there should be no heme-proteins in the sample. Such backgrounds have also been demonstrated to be the result of Mie-like scattering of both the source laser and the Raman bands themselves which enters the spectrometer as uncollimated stray light, together with ${ }_{60}$ variable contributions due to spot to spot sample morphology. ${ }^{37}$ Therefore, the application of spectral pre-processing or corrective methodologies was an integral step to ensure that robust and precise quantitative information may be extracted from the Raman dataset.

${ }_{65}$ Smoothing of the spectra was carried out using a Savitzky-Golay filter $^{40}$ to remove any spurious peaks and as dried plasma films generally produced spectra with a relatively high signal-to-noise 
ratio, a 3rd order, five data point window proved sufficient. Baseline offset correction was seen to be a crucial pre-processing step due to the large variation in background seen in the raw data. A polynomial baseline function was used and compared with an 5 iterative, linear-nodal correction (written in-house). For polynomial correction, band-free regions between wavenumber ranges of $400-630 \mathrm{~cm}^{-1}$ and $1502-1800 \mathrm{~cm}^{-1}$ were chosen, while the linear-nodal correction was executed in both automated node selection mode as well as manually, using the same 10 specified regions above. Vector normalisation is a standard method in which the total intensity of the spectral dataset is considered rather than individual peaks. This is achieved by calculating the sum of the

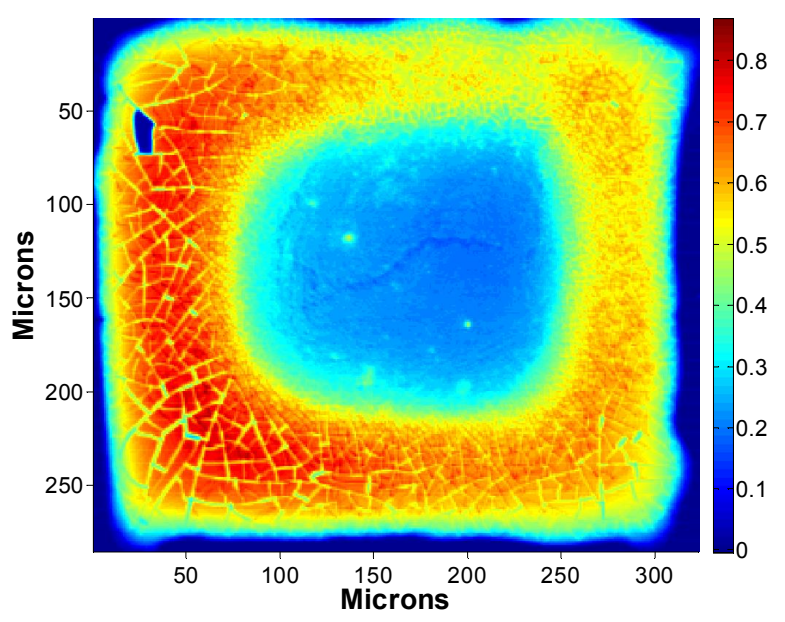

15 Figure 1. Average FTIR absorbance image showing inhomogeneous sample distribution. The scaled colour bar indicates that zero absorption occurs at 0 while maximum absorption occurs at 1.

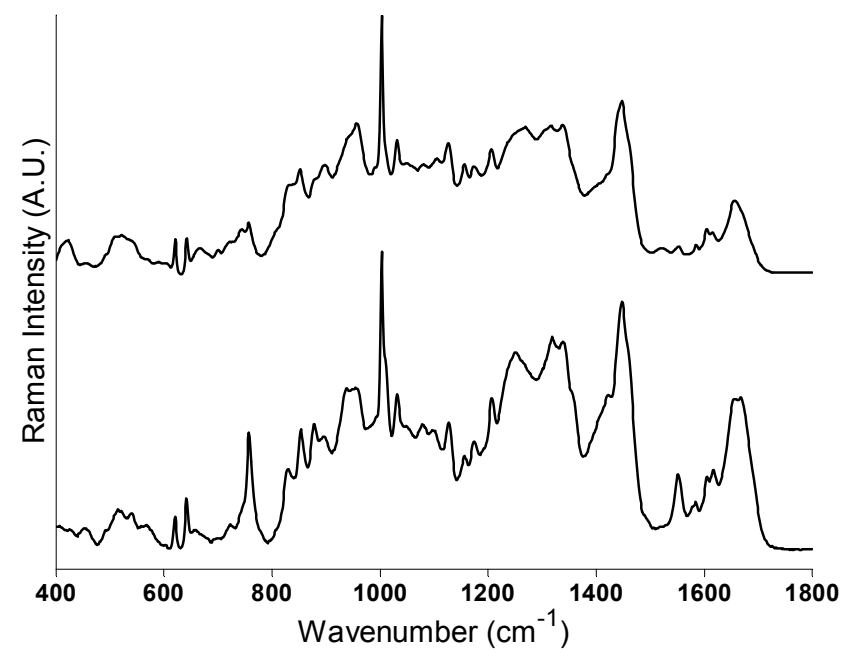

Figure 2. Typical spectrum of human blood plasma (top) and spectrum of 20 purified crystalline human fibrinogen (bottom).

squared intensity values of the spectrum and using the squared root of this sum as the normalisation constant. Each variable of a spectrum is then divided by this constant. Figure 4 shows vector normalisation applied to the averaged intersampled dataset. The

${ }_{25}$ data processing procedure has removed much of the variability of the raw spectra of Figure 3. All spectra were retained for the regression model to mimic as best as possible an automated sampling technique.

An alternative technique applied in order to remove
30 multiplicative effects from spectra was Extended Multiplicative Scattering Correction (EMSC). ${ }^{41}$ EMSC is a combination corrective algorithm dealing with both additive and multiplicative background effects of each sample spectrum originally developed for Fourier Transform Infrared spectroscopy.

\section{${ }_{35}$ PLS}

Two different datasets were used as the observed predictors (X) for the PLS algorithm; one utilised the entire original spectral dataset (100 spectra per sample), while the other was composed of an average, intersampled matrix to explore model efficiency 40 with a reduced, averaged dataset. The intersampling process was carried out through a four-point moving window average of the full dataset, reducing 100 spectra per sample to 25 averaged spectra. Intersampling, as we found, reduces the effect of outliers in the final result. Therefore, after random sorting of the datasets,

45 the training matrices were 2040 and 510 spectra $(60 \%$ split) for the full and averaged intersampled datasets respectively, with the remaining $40 \%$ split being designated as test matrices (1360 and

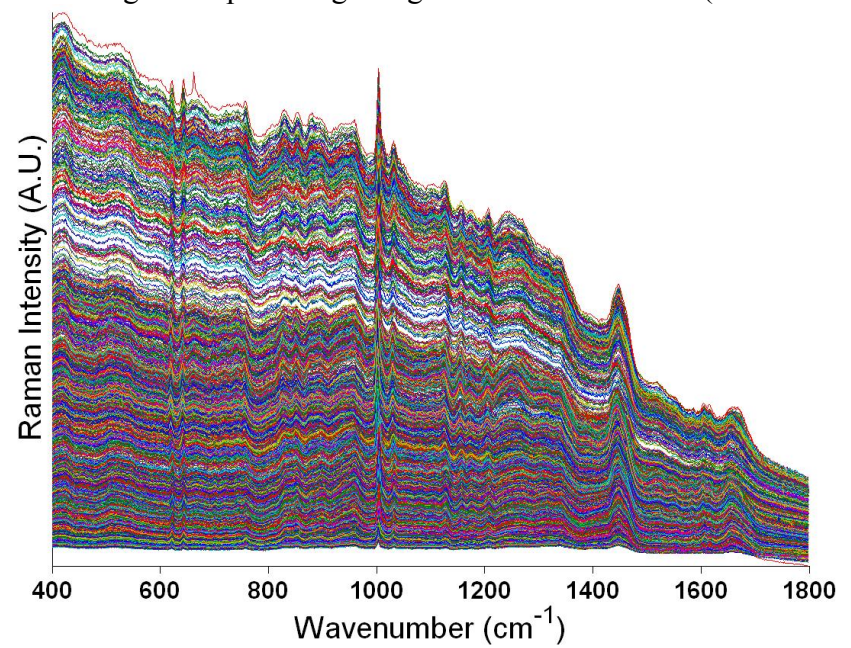

Figure 3. Raw spectra $(n=3400)$ obtained from all dried blood plasma samples.

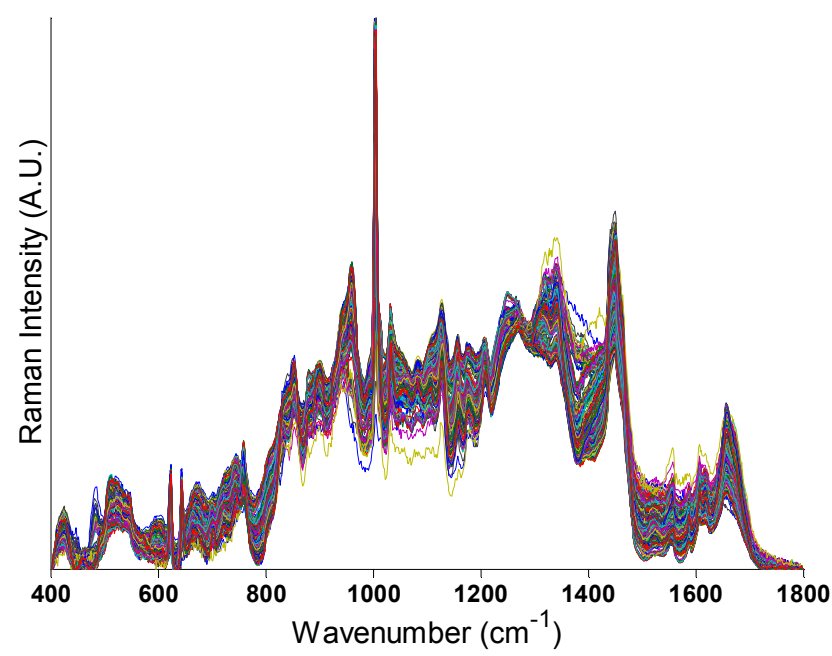

Figure 4. Average intersampled dataset $(n=850)$ after smoothing, automated linear-nodal baseline correction and vector normalisation.

340 spectra respectively). Out of the 100 iterations, Figure 5 55 shows an example of a single iteration of RMSEC and RMSECV against the number of latent variables from the PLS analysis showing, in this case the minimum RMSECV to occur at $L V_{\min }=$ 15. Once the model algorithms were sufficiently trained, validation of the model performance was carried out using the 
unseen, test matrices.

The fibrinogen concentrations from the 14 test sample sets were predicted by the constructed PLS model and evaluated against target values obtained by the Clauss fibrinogen assay. The 5 random nature of the cross-validation procedures resulted in the generation of different values of $L V_{\min }$ and hence prediction errors. Therefore, the model was trained and executed 100 separate times (with associated randomisation and splitting of the data on each run) to produce minimum, maximum and average 10 values of $L V_{\min }$, RMSEP and $\mathrm{R}^{2}$ with associated variances

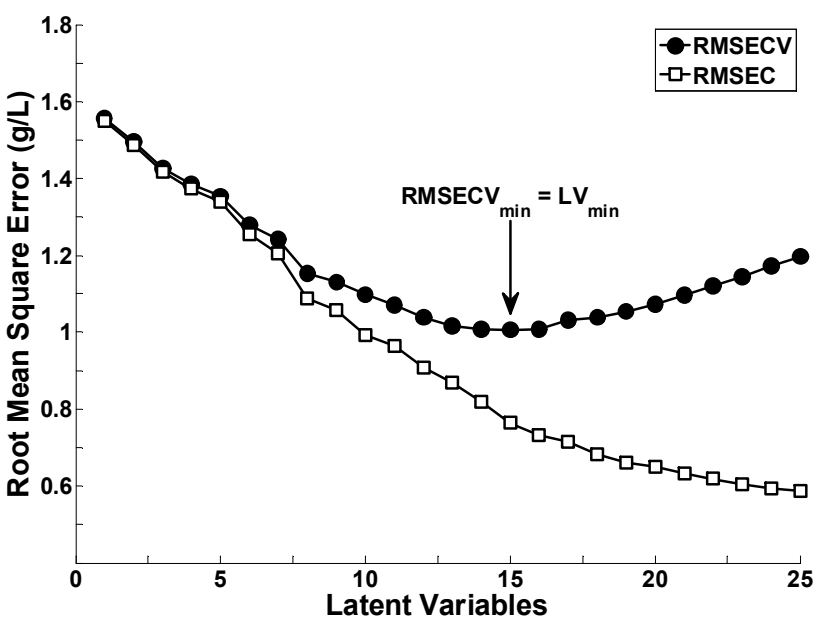

Figure 5. An example plot of the root mean square error of calibration (RMSEC) versus root mean square error of cross validation as a function of the number of latent variables. The optimum number of latent

15 variables corresponds to the minimum of RMSECV.

\section{DISCUSSION}

The predictive performances of the models show a close correlation between the concentrations predicted by Raman spectroscopy and target concentrations established by the Clauss 20 Fibrinogen assay. As can be seen from Figure 6, the wide scatter in predicted Fibrinogen concentrations using the full spectral dataset prevents an accurate classification of samples as either moderate but normal Fibrinogen concentrations $(1.7-4.0 \mathrm{~g} / \mathrm{L}$ as determined through clotting assays $)^{42}$ or high clinically 25 significant values (e.g. $7-9$ g/L). Furthermore, it is notable that the best fit regression does not extrapolate to the origin. The average intersampled dataset ( $n=25$ per sample) visually appears to contain less data scatter with less influence from outliers resulting in a stronger correlation coefficients (Figure 7), thus 30 improving prediction to $\leq 0.25 \mathrm{~g} / \mathrm{L}$ over the full dataset (Table 3). Extrapolation towards zero fibrinogen content was also more accurate within the averaged dataset. As previously mentioned, the inter-sampling process was carried out through a four-point moving window average of the full dataset, which effectively 35 reduced the influence of outliers. This may also imply that there are a threshold number of spectral replicates needed from a sample to accurately represent the biochemical content and to achieve quantification of a target constituent. However, on average, more latent variables or factors were required to explain 40 variations in the spectral data and target values using the averaged, intersampled dataset (mean $L V_{\min }=20$ ) than the full dataset (mean $L V_{\min }=15$ ).

In an attempt to improve the performance of the calibration model using the full dataset, a filtering technique was developed 45 for the removal of outliers. Firstly, PCA was employed to determine the principal component. The scores of each sample were then plotted onto a three-dimensional space and the mean centroid was determined from the resultant cluster. The sample(s) with the furthest calculated squared Euclidean distance from the 50 centroid are deemed outliers and omitted from the dataset. The furthest three, five and ten outliers were removed in this fashion. However, predictive errors were actually slightly increased (data not shown). This indicated that the selected outliers, which were
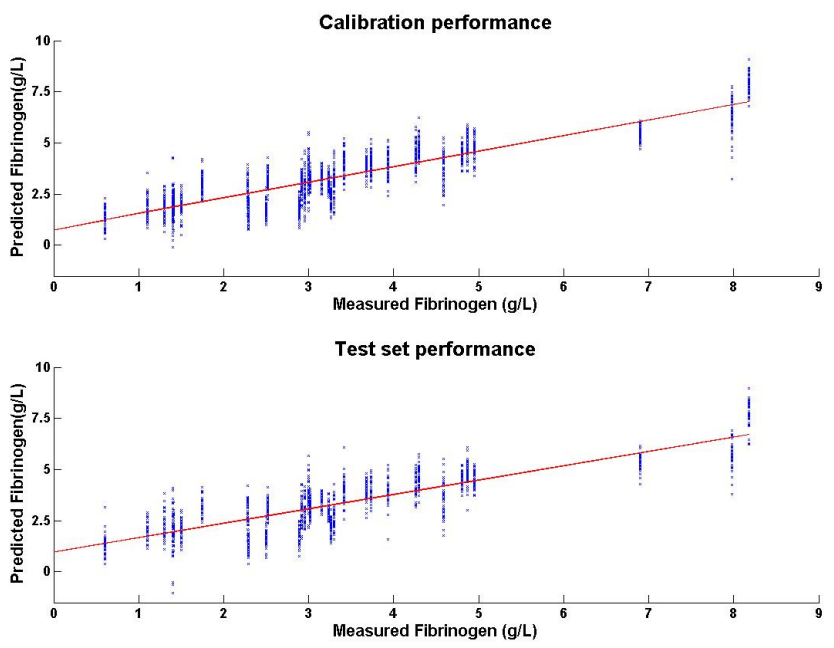

55 Figure 6. Performance of the PLS calibration model and subsequent test set prediction using the full spectral dataset.
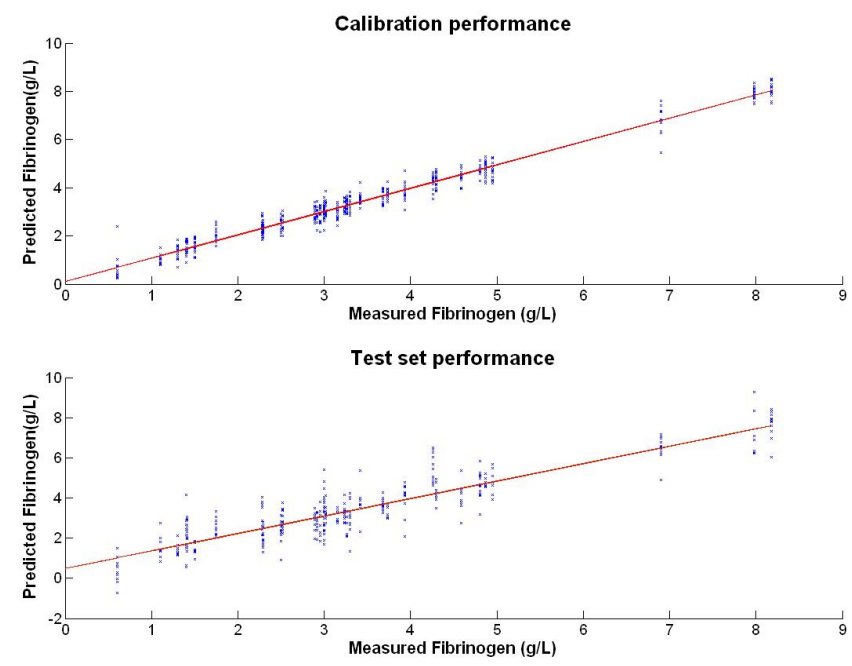

60 Figure 7. Performance of the PLS calibration model and subsequent test set prediction using the average intersampled spectral dataset.

deemed as such based on the loadings or intrasample variable relationships, still contributed in some way to the construction of the model.

${ }_{65}$ The two cross-validation procedures chosen produced slightly different prediction accuracies. While Leave-One-Out cross validation is a commonly used technique, it has a tendency to produce overly optimistic results for larger datasets $(n>20)$ and be prone to the replicate sample trap (replicates of the same 70 sample existing in both the training and the test set). ${ }^{43,}{ }^{44}$ The random sample selection cross validation method provides more 
realistic results and is more adaptable in that it may be used on many types of datasets with the advantage of avoiding the replicate sample trap through the use of a lower number of splits and an increased number of iterations. However, the random 5 nature of the latter method might lead to different researchers producing different PLS models of the same data. 
Table 3. Results of calibration and prediction PLS models using the test set. Values are averaged over 100 runs each with associated standard deviations. $(\mathrm{LOOCV}=$ Leave-One-Out Cross Validation, $\mathrm{RanCV}=$ Random Cross Validation $)$

\begin{tabular}{|c|c|c|c|c|}
\hline \multirow[b]{2}{*}{$\mathrm{n} L V_{\min }$} & \multicolumn{3}{|c|}{ Average intersampled Dataset ( $n=850,25$ spectra per sample) } & \multirow[b]{2}{*}{$R^{2}$ Prediction } \\
\hline & $\operatorname{RMSEC}(\mathrm{g} / \mathrm{L})$ & $\operatorname{RMSEP}(\mathrm{g} / \mathrm{L})$ & $R^{2}$ Calibration & \\
\hline \multicolumn{5}{|c|}{ Polynomial Baseline, Vector Normalised LOOCV } \\
\hline 20. & $0.38 \pm 0.04$ & $0.75 \pm 0.05$ & $0.98 \pm 5 \mathrm{E}-3$ & $0.90 \pm 0.01$ \\
\hline \multicolumn{5}{|c|}{ Polynomial Baseline, Vector Normalised RanCV } \\
\hline 20 & $0.41 \pm 0.03$ & $0.75 \pm 0.05$ & $0.97 \pm 4 \mathrm{E}-3$ & $0.90 \pm 0.01$ \\
\hline \multicolumn{5}{|c|}{ Polynomial Baseline, EMSC LOOCV } \\
\hline 20 & $0.40 \pm 0.08$ & $0.78 \pm 0.06$ & $0.97 \pm 0.01$ & $0.90 \pm 0.02$ \\
\hline \multicolumn{5}{|c|}{ Polynomial Baseline,EMSC RanCV } \\
\hline 18 & $0.44 \pm 0.07$ & $0.78 \pm 0.05$ & $0.97 \pm 0.01$ & $0.89 \pm 0.01$ \\
\hline \multicolumn{5}{|c|}{ Linear-Nodal, Vector Normalised LOOCV } \\
\hline 20 & $0.39 \pm 0.06$ & $0.72 \pm 0.05$ & $0.97 \pm 6 \mathrm{E}-3$ & $0.91 \pm 0.01$ \\
\hline \multicolumn{5}{|c|}{ Linear-Nodal, Vector Normalised RanCV } \\
\hline 20 & $0.42 \pm 0.02$ & $0.73 \pm 0.06$ & $0.97 \pm 4 \mathrm{E}-3$ & $0.91 \pm 0.01$ \\
\hline \multicolumn{5}{|c|}{ Linear-Nodal, EMSC LOOCV } \\
\hline 21 & $0.38 \pm 0.05$ & $0.73 \pm 0.05$ & $0.98 \pm 6 \mathrm{E}-3$ & $0.91 \pm 0.01$ \\
\hline \multicolumn{5}{|c|}{ Linear-Nodal, EMSC RanCV } \\
\hline 21 & $0.40 \pm 0.02$ & $0.73 \pm 0.06$ & $0.97 \pm 2 \mathrm{E}-3$ & $0.91 \pm 0.02$ \\
\hline \multicolumn{5}{|c|}{ Full Dataset $(n=3400,100$ spectra per sample $)$} \\
\hline $\mathrm{n} L V_{\min }$ & RMSEC & RMSEP & $R^{2}$ Calibration & $R^{2}$ Prediction \\
\hline \multicolumn{5}{|c|}{ Polynomial Baseline, Vector Normalised LOOCV } \\
\hline 17 & $0.78 \pm 0.03$ & $0.98 \pm 0.02$ & $0.89 \pm 8 \mathrm{E}-3$ & $0.83 \pm 8 \mathrm{E}-3$ \\
\hline \multicolumn{5}{|c|}{ Polynomial Baseline, Vector Normalised RanCV } \\
\hline 16 & $0.81 \pm 0.03$ & $0.99 \pm 0.02$ & $0.88 \pm 9 \mathrm{E}-3$ & $0.82 \pm 9 \mathrm{E}-3$ \\
\hline \multicolumn{5}{|c|}{ Polynomial Baseline, EMSC LOOCV } \\
\hline 17 & $0.80 \pm 0.02$ & $0.98 \pm 0.02$ & $0.89 \pm 7 \mathrm{E}-3$ & $0.83 \pm 9 \mathrm{E}-3$ \\
\hline \multicolumn{5}{|c|}{ Polynomial Baseline,EMSC RanCV } \\
\hline 16 & $0.82 \pm 0.02$ & $0.98 \pm 0.03$ & $0.88 \pm 6 \mathrm{E}-3$ & $0.83 \pm 9 \mathrm{E}-3$ \\
\hline \multicolumn{5}{|c|}{ Linear-Nodal, Vector Normalised LOOCV } \\
\hline 17 & $0.77 \pm 0.02$ & $0.97 \pm 0.02$ & $0.90 \pm 6 \mathrm{E}-3$ & $0.83 \pm 9 \mathrm{E}-3$ \\
\hline \multicolumn{5}{|c|}{ Linear-Nodal, Vector Normalised RanCV } \\
\hline 16 & $0.80 \pm 0.03$ & $0.98 \pm 0.02$ & $0.89 \pm 0.01$ & $0.83 \pm 8 \mathrm{E}-3$ \\
\hline \multicolumn{5}{|c|}{ Linear-Nodal, EMSC LOOCV } \\
\hline 18 & $0.78 \pm 0.02$ & $0.98 \pm 0.02$ & $0.89 \pm 6 \mathrm{E}-3$ & $0.83 \pm 9 \mathrm{E}-3$ \\
\hline \multicolumn{5}{|c|}{ Linear-Nodal, EMSC RanCV } \\
\hline 17 & $0.80 \pm 0.01$ & $0.98 \pm 0.02$ & $0.89 \pm 4 \mathrm{E}-3$ & $0.83 \pm 9 \mathrm{E}-3$ \\
\hline
\end{tabular}

Concerning the pre-processing of the spectral data before initiating model training, it is recognised that baseline correction should be carried out before any normalisation or corrective procedures to avoid incorporating baseline fluctuations into the 10 normalisation constant. ${ }^{45}$ Comparative results are shown regarding the use of an iterative linear-nodal and polynomial baseline correction algorithm (Table 3 - Supplementary Data). While the linear-nodal correction brought all spectra down to a common baseline (zero), the polynomial correction method 15 distributed spectra over a range of wavenumbers, hence the latter method producing $\approx 5 \%$ larger errors in prediction. With respect to the linear-nodal method, the choice between automated node selection and user-specified nodes saw the former perform better in terms of the prediction errors produced. This may be explained 20 in terms of the minute wavenumber shift of nodes in that they may not necessarily be located in the same region for all spectra during the correction. Hence the automated selection was a better choice to account for this.

For the correction of the inherent multiplicative effects of 25 scattering seen in terms of prediction errors, vector normalisation out-performed EMSC in the averaged intersampled dataset. While EMSC visually converged spectra more efficiently, it imposed a detrimental effect in failing to preserve key spectral features contributing to the training of a better quantitative 30 predictive model. To avoid inter-sample crossover effects, each sample set of spectra $(n=25$ or 100$)$ was either normalised or corrected with EMSC individually rather than applying the corrections over the entire dataset. As with baseline correction, the choice of method to correct for multiplicative effects appeared 35 to be more significant when applied to the averaged intersampled dataset.

To enable a measure of the model performance versus the stated precision of the Clauss assay in a clinical context, the coefficients of variation of the observed prediction errors $\left(\mathrm{CV}_{\mathrm{RMSE}}\right)$ were 40 calculated (6) and evaluated against the standard deviation of the reference values (calculated from the mean and relative coefficient of variation $\% \mathrm{CV}(7)$ ) provided by the manufacturer (Table 1).

$$
C V_{R M S E}=\frac{R M S E}{\bar{x}}
$$




$$
\% C V=\frac{\sigma}{\bar{x}} \times 100
$$

Where $\boldsymbol{\sigma}=$ standard deviation, $\overline{\mathbf{x}}=$ mean

The precision of the reference assay is described by a standard deviation of 0.17 and 0.14 for day-to-day and test-to-test $s$ respectively, as specified by the manufacturer. This is compared to a $\mathrm{CV}_{\mathrm{RMSE}}$ of 0.19 (best) and 0.21 (mean) as achieved by Raman spectroscopic measurements. These figures suggest that the Clauss assay slightly outperforms the Raman spectroscopic model with regards to precision or reproducibility in the 10 measurement of fibrinogen concentration. Ignoring the influence of outliers, the model shows a calibration accuracy of approximately $1 \mathrm{~g} / \mathrm{L}$ and $2 \mathrm{~g} / \mathrm{L}$ for the test set. Nevertheless, the case is presented for the technique to be a potential analytical tool for a frequently required measurement. It is noted that the sample 15 preparation was somewhat crude, and that the measurements were made on the thinnest regions of the sample. Improved sample preparation by for example spin coating could significantly improve signal to noise and spectral background. Ultimately, in situ measurements in wet samples would be 20 desirable.

Attention has to be drawn to the patient samples, which featured almost $50 \%$ of the values below $3 \mathrm{~g} / \mathrm{L}$ and only three values above $5 \mathrm{~g} / \mathrm{L}$, this reflects the normal healthy range of $2.2-4.3$ $\mathrm{g} / \mathrm{L}$. However, this unbalance not only affected the correlation but 25 also the errors associated with the constructed model. However, the choice was made to include all samples without dilution to maintain the reagent-free nature of the study, ensuring a robust comparison of the technique to the gold-standard assay. As this is a pilot study to test the sensitivity of Raman spectroscopy, a 30 larger sample set of blood samples was not pursued. Of greater importance was to explore and establish the range of fibrinogen concentrations to construct a robust model. It is noted that calibration against a limited gold standard is a process that is in itself obviously limited. Ultimately, a cross validation against 35 more precise proteomic techniques would be required.

\section{Conclusions}

This study investigated the potential of Raman spectroscopy to quantify the Factor I fibrinogen protein in undiluted, dried patient blood plasma samples. The use of dried blood plasma forgoes the 40 issues related to background water spectral contributions, scattering, heating and biohazard safety. Also, the small volume $(10 \mu \mathrm{L})$ of plasma needed for analysis means the majority of the blood sample is retained for parallel tests. Partial Least Squares regression was employed to construct and train a calibration 45 model using Raman spectra obtained from plasma samples as fitted to target fibrinogen values obtained from the Clauss assay. It was shown that the various spectral pre-processing methods chosen in this study affected model training performance and resultant prediction errors. The best predictive result $(0.72 \pm 0.05$ ${ }_{50} \mathrm{~g} / \mathrm{L}$ ) came with the use of an averaged, intersampled dataset that had been smoothed using a Savitzky-Golay filter, baseline corrected using an automated linear-nodal algorithm and vector normalised. While the latter combination of methods worked best in this particular study, given a different analyte and/or ${ }_{55}$ instrumental setup the pre-processing techniques would no doubt be suitably modified. Although pre-processing remains an essential part of Raman spectroscopy, unwanted variability may be introduced through over manipulation of spectral data, which is especially applicable in sensitive quantitative analysis. Thus ${ }_{60}$ the basic rule of thumb is to ensure to preserve spectral integrity through the use of only the minimum amount of pre-processing needed. While the accuracy of Raman spectroscopy failed to match that of the gold-standard assay in this study, a more balanced dataset or calibration using certified fibrinogen ${ }_{65}$ standards and controls would possibly improve performance towards clinical application. However, the number of spectral replicates and hence acquisition times needs to be reduced for the purposes of daily routine analysis. It is noted that calibration against a limited gold standard is a process which is in itself 70 obviously limited. Ultimately, a cross validation against more precise proteomic techniques would be required. However, the study demonstrates that, as a quantitative tool, spontaneous, unenhanced Raman spectroscopy is a highly reproducible technique in the detection of single components in a complex 75 biological matrix.

\section{Acknowledgements}

This work was funded by the Department of Education and Science Technological Sector Research Strand III programme and enabled through the National Biophotonics and Imaging

${ }_{80}$ Platform, Ireland, and the Integrated NanoScience Platform for Ireland, both funded by the Irish Government's Programme for Research in Third Level Institutions, Cycle 4, National Development Plan 2007-2013.

\section{Notes and references}

$85 *$ Corresponding author.

${ }^{a}$ RESC, Focas Research Institute, Dublin Institute of Technology, Kevin Street,Dublin 8, Ireland.E-mail: kelvin.poon@dit.ie.

${ }^{b}$ Focas Research Institute, Dublin Institute of Technology, Kevin Street, Dublin 8, Ireland

$90{ }^{c}$ School of Biological Sciences, Dublin Institute of Technology, Kevin Street, Dublin 8, Ireland.

1. B. Holm and H. C. Godal, Thrombosis Research, 1984, 35, 279-290.

2. A. Sonel, B. M. Sasseen, N. Fineberg, N. Bang and R. L. Wilensky, $95 \quad$ Circulation, 2000, 102, 1107-1113.

3. J. Heinrich, L. Balleisen, H. Schulte, G. Assmann and J. Vandeloo, Arterioscler. Thromb., 1994, 14, 54-59.

4. I. J. Mackie, S. Kitchen, S. J. Machin and G. D. O. Lowe, British Journal of Haematology, 2003, 121, 396-404.

100 5. S. Polterauer, V. Seebacher, K. Hefler-Frischmuth, C. Grimm, G. Heinze, C. Tempfer, A. Reinthaller and L. Hefler, American Journal of Obstetrics and Gynecology, 2009, 200, 647.e641647.e647.

6. A. Clauss, Acta Haematologica, 1957, 17, 237-246.

105 7. O. D. Ratnoff and C. Menzie, J. Lab. Clin. Med., 1951, 37, 316-320.

8. U. Becker, K. Bartl and A. W. Wahlefeld, Thrombosis Research, 1984, 35, 475-484.

9. T. Exner, J. Burridge, P. Power and K. A. Rickard, American Journal of Clinical Pathology, 1979, 71, 521-527.

110 10. F. H. Schulz, Acta Hepatol, 1955, 3, 306-310.

11. UK National External Quality Assessment Scheme (NEQAS), Blood Coagulation, Sheffield, UK, 2000.

12. B. Holm, F. Brosstad, P. Kierulf and H. C. Godal, Thrombosis Research, 1985, 39, 595-606. 
13. Medical Devices Agency, Evaluation Report: Fibrinogen Standards and Reference preparations, 1999.

14. Medical Devices Agency, Evaluation Report: Fibrinogen Assay Reagents and Methods, 2000.

5 15. I. J. Mackie, A. S. Lawrie, S. Kitchen, P. J. Gaffney, D. Howarth, G. D. O. Lowe, J. Martin, G. Purdy, P. Rigsby and A. Rumley, Thromb. Haemost., 2002, 87, 997-1005.

16. J. W. Chan, D. S. Taylor, T. Zwerdling, S. M. Lane, K. Ihara and T. Huser, Biophysical Journal, 2006, 90, 648-656.

10 17. P. Crow, B. Barrass, C. Kendall, M. Hart-Prieto, M. Wright, R. Persad and N. Stone, Br J Cancer, 2005, 92, 2166-2170.

18. P. R. T. Jess, D. D. W. Smith, M. Mazilu, K. Dholakia, A. C. Riches and C. S. Herrington, International Journal of Cancer, 2007, 121, 2723-2728.

15 19. H. Abramczyk, I. Placek, B. Brożek-Płuska, K. Kurczewski, Z. Morawiec and M. Tazbir, Spectroscopy, 2008, 22, 113-121.

20. M. J. Baker, E. Gazi, M. D. Brown, J. H. Shanks, N. W. Clarke and P. Gardner, Journal of Biophotonics, 2009, 2, 104-113.

21. J. Hutchings, C. Kendall, B. Smith, N. Shepherd, H. Barr and N.

20

22. C. A. Lieber and A. Mahadevan-Jansen, Appl. Spectrosc., 2003, 57, 1363-1367.

23. F. M. Lyng, E. Ó. Faoláin, J. Conroy, A. D. Meade, P. Knief, B. Duffy, M. B. Hunter, J. M. Byrne, P. Kelehan and H. J. Byrne,

25 Experimental and Molecular Pathology, 2007, 82, 121-129.

24. D. Rohleder, G. Kocherscheidt, K. Gerber, W. Kiefer, W. Kohler, J. Mocks and W. Petrich, J. Biomed. Opt., 2005, 10, 10.

25. J. Y. Qu, B. C. Wilson and D. Suria, Appl. Opt., 1999, 38, 54915498.

30 26. W. Petrich, A. Staib, M. Otto and R. L. Somorjai, Vib. Spectrosc., 2002, 28, 117-129.

27. H. W. Gerhard, B. Dirk, H. Hans-Peter, K. Hans-Juergen and M. Reinhold, 1998.

28. A. J. Berger, T.-W. Koo, I. Itzkan, G. Horowitz and M. S. Feld, Appl. 35 Opt., 1999, 38, 2916-2926.

29. H. M. Heise and A. Bittner, Fresenius' Journal of Analytical Chemistry, 1998, 362, 141-147.

30. S. Low-Ying, R. A. Shaw, M. Leroux and H. H. Mantsch, Vib. Spectrosc., 2002, 28, 111-116.

40 31. D. Rohleder, W. Kiefer and W. Petrich, Analyst, 2004, 129, 906-911.

32. A. Enejder, M. K., T. Scecina, G., J. Oh, M. Hunter, W.-C. Shih, S. Sasic, G. Horowitz, L. and M. Feld, S., J. Biomed. Opt., 2005, 10, 031114.

33. A. M. K. Enejder, T. W. Koo, J. Oh, M. Hunter, S. Sasic, M. S. Feld

45 and G. L. Horowitz, Optics Letters, 2002, 27, 2004-2006.

34. A. D. Meade, C. Clarke, H. J. Byrne and F. M. Lyng, Radiat. Res., 2010, 173, 225-237.

35. H. Nawaz, F. Bonnier, P. Knief, O. Howe, F. M. Lyng, A. D. Meade and H. J. Byrne, Analyst, 2010.

50 36. S. de Jong, Chemometrics and Intelligent Laboratory Systems, 1993, 18, 251-263.

37. F. Bonnier, A. Mehmood, P. Knief, A. D. Meade, W. Hornebeck, H. A. Lambkin, K. Flynn, V. McDonagh, C. Healy, T. C. Lee, F. M. Lyng and H. J. Byme, J. Raman Spectrosc., 2010.

55 38. M. Chaplin, 2011.

39. F. Bonnier, A. Mehmood, P. Knief, A. D. Meade, W. Hornebeck, H. Lambkin, K. Flynn, V. McDonagh, C. Healy, T. C. Lee, F. M.
Lyng and H. J. Byrne, J. Raman Spectrosc., 2011, 42, 888896.

60 40. A. Savitzky and M. J. E. Golay, Anal. Chem., 1964, 36, 1627-\&.

41. A. Kohler, C. Kirschner, A. Oust and H. Martens, Appl. Spectrosc., 2005, 59, 707-716.

42. M. P. M. De Maat, G. D. O. Lowe and F. Haverkate, in Laboratory Techniques in Thrombosis - A Manual, Kluwer Academic Publishers, Boston 2nd edn., 1999, pp. 79-88.

43. L. Eriksson, E. Johansson, M. Müller and S. Wold, Journal of Chemometrics, 2000, 14, 599-616.

44. H. A. Martens and P. Dardenne, Chemometrics and Intelligent Laboratory Systems, 1998, 44, 99-121.

70 45. N. K. Afseth, V. H. Segtnan and J. P. Wold, Appl. Spectrosc., 2006, 60, 1358-1367. 